\title{
Spin pumping and magnetization dynamics in metallic multilayers
}

\author{
Yaroslav Tserkovnyak and Arne Brataas* \\ Lyman Laboratory of Physics, Harvard University, Cambridge, Massachusetts 02138 \\ Gerrit E. W. Bauer \\ Department of NanoScience, Delft University of Technology, 2628 CJ Delft, The Netherlands
}

(Received 6 August 2002; published 5 December 2002)

\begin{abstract}
We study the magnetization dynamics in thin ferromagnetic films and small ferromagnetic particles in contact with paramagnetic conductors. A moving magnetization vector causes "pumping" of spins into adjacent nonmagnetic layers. This spin transfer affects the magnetization dynamics similar to the Landau-LifshitzGilbert phenomenology. The additional Gilbert damping is significant for small ferromagnets, when the nonmagnetic layers efficiently relax the injected spins, but the effect is reduced when a spin accumulation build-up in the normal metal opposes the spin pumping. The damping enhancement is governed by (and, in turn, can be used to measure) the mixing conductance or spin-torque parameter of the ferromagnet-normal-metal interface. Our theoretical findings are confirmed by agreement with recent experiments in a variety of multilayer systems.
\end{abstract}

DOI: 10.1103/PhysRevB.66.224403 PACS number(s): 72.25.Mk, 75.70.Cn, 76.50.+g, 76.60.Es

\section{INTRODUCTION}

Spin-polarized transport through magnetic multilayers is the physical origin of many exciting phenomena such as giant magnetoresistance and spin-current-induced magnetization reversal. ${ }^{1-3}$ It has attracted attention in the basic physics community and industry over the last decades, but there are still open fundamental questions. So far, the main research activity has been focused on the dc transport properties of these systems.

Ac magnetotransport has attracted considerably less attention than its dc counterpart. In a recent paper, ${ }^{4}$ we reported a novel mechanism by which a precessing ferromagnet "pumps" a spin current into adjacent nonmagnetic conductors proportional to the precession frequency, using a formalism analogous to that for the adiabatic pumping of charges in mesoscopic systems. ${ }^{5}$ We showed that spin pumping profoundly affects the dynamics of nanoscale ferromagnets and thin films, by renormalizing fundamental parameters such as the gyromagnetic ratio and Gilbert damping parameter, in agreement with experiments. ${ }^{6}$

The switching characteristics of a magnetic system depends in an essential way on the Gilbert damping constant $\alpha$. In magnetic field-induced switching processes, for example, $\alpha$ governs the technologically important magnetization reversal time of ferromagnetic particles. Its typical intrinsic value $^{7} \alpha_{0} \lesssim 10^{-2}$ for transition metal ferromagnets is smaller than its optimal value of $\alpha \gtrsim 10^{-1}$ for the fastest switching rates. $^{8}$ The present mechanism allows engineering of the damping constant by adding passive nonmagnetic components to the system and/or adjusting the geometry to control spin flow and relaxation rates described in this paper, thus helping to create high-speed magnetoelectronic devices. Also, in spin-current-induced magnetization reversal, the critical switching current is proportional to $\alpha$. $^{3}$

For some time it has been understood that a ferromagnetnormal-metal $(F-N)$ interface leads to a dynamical coupling between the ferromagnetic magnetization and the spins of the conduction-band electrons in the normal metal. ${ }^{2,3,9-12}$
More recently, several theoretical frameworks were put forward proposing a mechanism for magnetization damping due to $F-N$ interfacial processes. ${ }^{4,10,13}$ This $F-N$ coupling becomes important in the limit of ultrathin $(\$ 10 \mathrm{~nm})$ ferromagnetic films and can lead to a sizable enhancement of the damping constant.

Our theory is based on a new physical picture, according to which the ferromagnetic damping can be understood as an adiabatic pumping of spins into the adjacent normal metals. ${ }^{4}$ This spin transfer is governed by the reflection and transmission matrices of the system, analogous to the scattering theory of transport and interlayer exchange coupling. The microscopic expression for the enhanced Gilbert damping and the renormalized gyromagnetic ratio can be calculated by simple models or by first-principles band-structure calculations without adjustable parameters. The present theory therefore allows quantitative predictions for the magnetization damping in hybrid systems that can be tested by experiments.

The Gilbert damping constant in thin ferromagnetic films has been experimentally studied ${ }^{6,14-17}$ by measuring ferromagnetic-resonance (FMR) linewidths. In the regime of ultrathin ferromagnetic films, $\alpha$ was in some cases found to be quite large in comparison with the bulk value $\alpha_{0}$, and sensitively depending on the substrate and capping layer materials. For example, when a 20 - $\AA$-thick permalloy (Py) film was sandwiched between two Pt layers, its damping was found to be $\alpha \sim 10^{-1}$, but recovered its bulk value $\alpha$ $\sim 10^{-2}$ with a $\mathrm{Cu}$ buffer and cap. ${ }^{6}$ Heinrich et al. ${ }^{14}$ observed an enhanced damping of $\lesssim 20$ - $\AA$-thick Fe films when they were grown on Ag bulk substrates but no significant change in the damping constant was seen for films grown on GaAs even when the film thickness was reduced down to several atomic monolayers. ${ }^{18}$ We will demonstrate here that our theory explains all these experimental findings well.

Previously, we studied the situation when the normalmetal layers adjacent to the ferromagnetic films are perfect spin sinks, so that the spin accumulation in the normal metal vanishes. ${ }^{4}$ Here this theory is generalized to consider the spin 
accumulation which enables us to explain experimental findings for various $F-N$ systems $s^{6,14,16}$ in a unified framework based on the spin-pumping picture.

The paper is organized as follow. In Sec. II and the Appendix, the basic formalism of the adiabatic spin-pump theory ${ }^{4}$ is derived using a scattering-matrix approach, and an alternative derivation is given for finite systems, which is based on the conservation of energy and angular momentum. In Secs. III and IV, we solve the diffusion equation to describe transport of injected spins in single and composite normal-metal layers. The spin loss due to spin-orbit or other spin-flip processes is accounted for, leading to an overall damping of the ferromagnetic magnetization precession. In particular, we use the theory to analyze the representative case of Gilbert damping in Py-Pt, $\mathrm{Py}-\mathrm{Cu}$, and $\mathrm{Py}-\mathrm{Cu}-\mathrm{Pt}$ hybrids, showing an excellent agreement between our theory and the experimental results. ${ }^{6,16}$ The last Sec. V is devoted to discussions and conclusions.

\section{PRECESSION-INDUCED SPIN PUMPING}

Consider an $N-F-N$ junction as in Fig. 1 and Fig. 6 in the Appendix. Without a voltage bias, no spin or charge currents flow when the magnetization of the ferromagnet is constant in time. When the magnetization direction starts precessing (as, e.g., under the influence of an applied magnetic field), a spin current $\mathbf{I}_{s}^{\text {pump }}$ is pumped out of the ferromagnet. ${ }^{4}$ This current into a given $N$ layer depends on the complex-valued parameter $A \equiv A_{r}+i A_{i}$ (the "spin-pumping conductance") by

$$
\mathbf{I}_{s}^{\text {pump }}=\frac{\hbar}{4 \pi}\left(A_{r} \mathbf{m} \times \frac{d \mathbf{m}}{d t}-A_{i} \frac{d \mathbf{m}}{d t}\right) .
$$

Here, the time-dependent order parameter of the ferromagnet is a unit vector $\mathbf{m}(t)$, assuming a monodomain magnet with a spatially uniform magnetization at all times. A detailed derivation of Eq. (1) based on the scattering-matrix theory of transport is presented in the Appendix. $A=g^{\uparrow \downarrow}-t^{\uparrow \downarrow}$ depends on the scattering matrix of the ferromagnetic film since

$$
g^{\sigma \sigma^{\prime}} \equiv \sum_{m n}\left[\delta_{m n}-r_{m n}^{\sigma}\left(r_{m n}^{\sigma^{\prime}}\right)^{*}\right]
$$

is the dimensionless dc conductance matrix ${ }^{11,12}$ and

$$
t^{\uparrow \downarrow} \equiv \sum_{m n} t_{m n}^{\prime \uparrow}\left(t_{m n}^{\prime \downarrow}\right)^{*} .
$$

Here (see Fig. 6), $r_{m n}^{\uparrow}\left(r_{m n}^{\downarrow}\right)$ is a reflection coefficient for spin-up (spin-down) electrons on the normal-metal side and $t_{m n}^{\prime \uparrow}\left(t_{m n}^{\prime \downarrow}\right)$ is a transmission coefficient for spin-up (spindown) electrons across the ferromagnetic film from the opposite reservoir into the normal-metal layer, where $m$ and $n$ label the transverse modes at the Fermi energy in the normalmetal films. Note that the magnetization can take arbitrary directions; in particular, $\mathbf{m}(t)$ may be far away from its equilibrium value. In such a case, the scattering matrix itself can depend on the orientation of the magnetization, and one has to use $A(\mathbf{m})$ in Eq. (1).

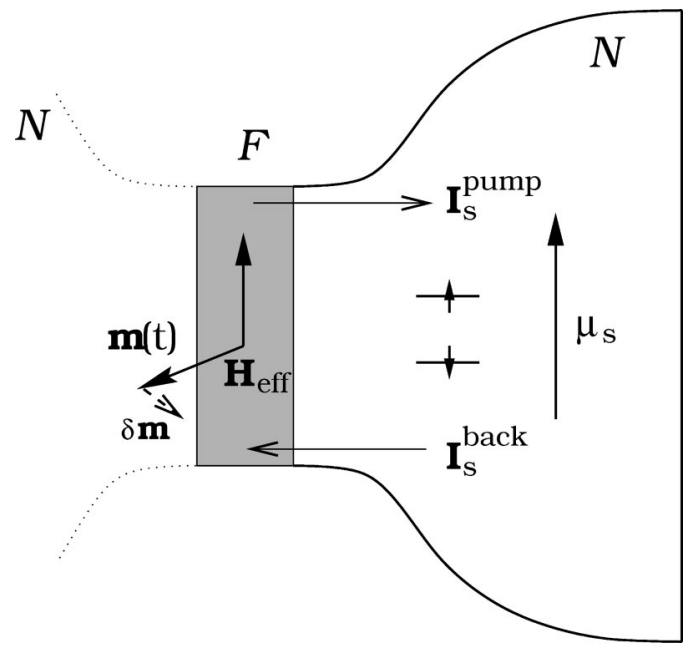

FIG. 1. A ferromagnetic film $F$ sandwiched between two nonmagnetic reservoirs $N$. For simplicity of the discussion in this section, we mainly focus on the dynamics in one (right) reservoir while suppressing the other (left), e.g., assuming it is insulating. The spinpumping current $\mathbf{I}_{s}^{\text {pump }}$ and the spin accumulation $\mu_{s}$ in the right reservoir can be found by conservation of energy, angular momentum, and by applying circuit theory to the steady state $\mathbf{I}_{s}^{\text {pump }}$ $=\mathbf{I}_{s}^{\text {back }}$.

When the ferromagnetic film is thicker than its transverse spin-coherence length $d>\pi /\left(k_{\mathrm{F}}^{\uparrow}-k_{\mathrm{F}}^{\downarrow}\right)$, where $k_{\mathrm{F}}^{\uparrow(\downarrow)}$ are the spin-dependent Fermi wave vectors, $t^{\uparrow \downarrow}$ vanishes, ${ }^{20}$ the spin pumping through a given $F-N$ interface is governed entirely by the interfacial mixing conductance $A=g^{\uparrow \downarrow} \equiv g_{r}^{\uparrow \downarrow}+i g_{i}^{\uparrow \downarrow}$, and we can consider only one of the two interfaces. This is the regime we are focusing on in this paper. Note that the conductance matrix $g^{\sigma \sigma^{\prime}}$ defined in Eq. (2) has to be renormalized for highly transparent interfaces in columnar geometries (by properly subtracting Sharvin-resistance contributions from the inverse conductance parameters), as discussed in Ref. 19.

As shown before, ${ }^{4}$ the spin current [Eq. (1)] leads to a damping of the ferromagnetic precession, resulting in a faster alignment of the magnetization with the (effective) applied magnetic field $\mathbf{H}_{\mathrm{eff}}$. In the derivation by the time-dependent scattering theory, the pumped spins are entirely absorbed by the attached ideal reservoirs. In the following, it is shown that Eq. (1) can be also derived for a finite system by observing that the enhanced rate of damping is accompanied by an energy flow out of the ferromagnet, until a steady state is established in the combined $F-N$ system. For simplicity, assume a magnetization which at time $t$ starts rotating around the vector of the magnetic field $\mathbf{m}(t) \perp \mathbf{H}_{\text {eff }}$. In a short interval of time $\delta t$, it slowly (i.e., adiabatically) changes to $\mathbf{m}(t$ $+\delta t)=\mathbf{m}(t)+\delta \mathbf{m}$. In the presence of a large but finite nonmagnetic reservoir without any spin-flip scattering attached to one side of the ferromagnet, this process can be expected to induce a (small) nonvanishing spin accumulation

$$
\boldsymbol{\mu}_{s} \equiv \int d \epsilon \operatorname{Tr}[\hat{\boldsymbol{\sigma}} \hat{f}(\epsilon)]
$$


where $\hat{\boldsymbol{\sigma}}$ is the Pauli matrix vector and $\hat{f}(\boldsymbol{\epsilon})$ is the $2 \times 2$ matrix distribution function at a given energy $\epsilon$ in the reservoir. ${ }^{11}$ For a slow enough variation of $\mathbf{m}(t)$, this nonequilibrium spin imbalance must flow back into the ferromagnet, canceling any spin current generated by the magnetization rotation, since, due to the adiabatic assumption, the system is always in a steady state.

Let us assume for the moment that the spins are accumulated in the reservoir along the magnetic field $\boldsymbol{\mu}_{s} \| \mathbf{H}_{\text {eff }}$. Flow of $N_{s}$ spins into the normal metal transfers energy $\Delta E_{N}$ $=N_{s} \mu_{s} / 2$ and angular-momentum $\Delta L_{N}=N_{s} \hbar / 2$ (directed along $\left.\mathbf{H}_{\text {eff }}\right)$. By the conservation laws, $\Delta E_{F}=-\Delta E_{N}$ and $\Delta L_{F}=-\Delta L_{N}$, for the corresponding values in the ferromagnet. Using the magnetic energy $\Delta E_{F}=\gamma \Delta L_{F} H_{\text {eff }}$, where $\gamma$ is the absolute gyromagnetic ratio of the ferromagnet, we then find that $N_{s} \mu_{s} / 2=\gamma N_{s}(\hbar / 2) H_{\text {eff }}$. It then follows that $\mu_{s}$ $=\hbar \gamma H_{\text {eff }}=\hbar \omega$, where $\omega=\gamma H_{\text {eff }}$ is the Larmor frequency of precession in the effective field: The spin-up and spin-down chemical potentials in the normal metal are split by $\mu_{s}$ $=\hbar \omega$, the energy corresponding to the frequency of the perturbation. For a finite angle $\theta$ between $\boldsymbol{\mu}_{s}$ and $\mathbf{H}_{\text {eff }}$, the same reasoning would lead to $\mu_{s}=\hbar \omega \cos \theta$, which is smaller than the "energy boost" $\hbar \omega$ of the time-dependent perturbation, thus justifying our initial guess.

We can employ magnetoelectronic circuit theory ${ }^{11}$ to derive an expression for the backflow of spin current $\mathbf{I}_{s}^{\text {back }}$ which, as argued above, has to be equal to the pumping current $\mathbf{I}_{s}^{\text {pump }}=\mathbf{I}_{s}^{\text {back }}$ :

$$
\begin{aligned}
\mathbf{I}_{s}^{\text {back }} & =\frac{1}{2 \pi}\left(g_{r}^{\uparrow \downarrow} \boldsymbol{\mu}_{s}+g_{i}^{\uparrow \downarrow} \mathbf{m} \times \boldsymbol{\mu}_{s}\right) \\
& =\frac{\hbar}{4 \pi}\left(g_{r}^{\uparrow \downarrow} \mathbf{m} \times \frac{d \mathbf{m}}{d t}-g_{i}^{\uparrow \downarrow} \frac{d \mathbf{m}}{d t}\right) .
\end{aligned}
$$

Here, we used $\mu_{s}=\hbar \omega$ and $\boldsymbol{\mu}_{s} \perp \mathbf{m}$, since by the conservation of angular momentum, the spin transfer is proportional to the change in the direction $\delta \mathbf{m} \perp \mathbf{m}$. We thus recover Eq. (1) for the case of a single and finite reservoir. It is easy to repeat the proof for an arbitrary initial alignment of $\mathbf{m}(t)$ with $\mathbf{H}_{\text {eff }}$. Furthermore, a straightforward generalization of this discussion to the case of the $N-F-N$ sandwich structure recovers our previous result [Eq. (1)].

The expressions for the adiabatic spin pumping are not the whole story, since spin-flip scattering is an important fact of life in magnetoelectronics. In Ref. 4, we only considered the extreme situation where the normal-metal layer is a perfect spin sink, so that all spins injected by $\mathbf{I}_{s}^{\text {pump }}$ relax by spin-flip processes or leave the system; the total spin current through the contact was, therefore, approximated by $\mathbf{I}_{s}$ $\approx \mathbf{I}_{s}^{\text {pump }}$ and $\mathbf{I}_{s}^{\text {back }} \approx 0$. Here, we generalize that treatment to self-consistently take into account the spin build-up in the normal metal at dynamic equilibrium. We then find the contribution to $\mathbf{I}_{s}$ due to the spin-accumulation-driven current $\mathbf{I}_{s}^{\text {back }}$ back into the ferromagnet:

$$
\mathbf{I}_{s}=\mathbf{I}_{s}^{\text {pump }}-\mathbf{I}_{s}^{\text {back }},
$$

which vanishes in the absence of spin-flip scattering.

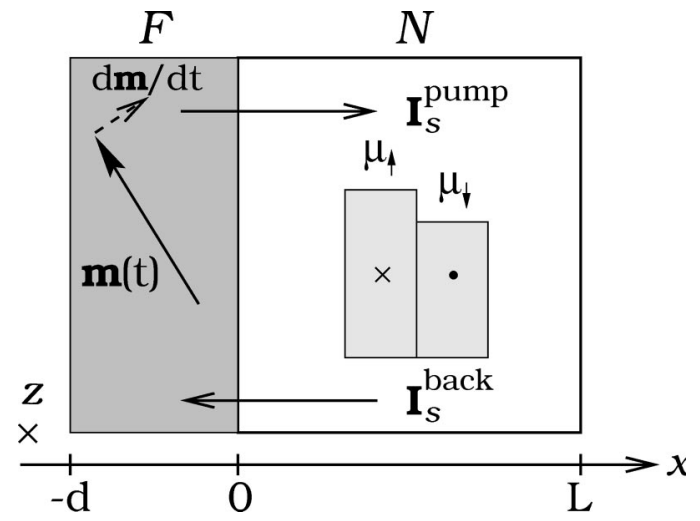

FIG. 2. Schematic view of the $F-N$ bilayer. Precession of the magnetization direction $\mathbf{m}(t)$ of the ferromagnet $F$ pumps spins into the adjacent normal-metal layer $N$ by inducing a spin current $\mathbf{I}_{s}^{\text {pump }}$. This leads to a build-up of the normal-metal spin accumulation which either relaxes by spin-flip scattering or flows back into the ferromagnet as $\mathbf{I}_{s}^{\text {back }}$. In contrast to Fig. 1, the $N$ layer here is not an ideal reservoir but rather a film of the same cross section as the magnetic layer $F$; the spin accumulation is position $(x)$ dependent.

The spin current out of the ferromagnet carries angular momentum perpendicular to the magnetization direction. By conservation of angular momentum, the spins ejected by $\mathbf{I}_{s}$ correspond to a torque $\boldsymbol{\tau}=-\mathbf{I}_{s}$ on the ferromagnet. If possible interfacial spin-flip processes are disregarded, the torque $\tau$ is entirely transferred to the coherent magnetization precession. The dynamics of the ferromagnet can then be described by a generalized Landau-Lifshitz-Gilbert (LLG) equation $^{21,2}$

$$
\frac{d \mathbf{m}}{d t}=-\gamma \mathbf{m} \times \mathbf{H}_{\mathrm{eff}}+\alpha_{0} \mathbf{m} \times \frac{d \mathbf{m}}{d t}+\frac{\gamma}{M_{s} V} \mathbf{I}_{s},
$$

where $\alpha_{0}$ is the dimensionless bulk Gilbert damping constant, $M_{s}$ is the saturation magnetization of the ferromagnet, and $V$ is its volume. The intrinsic bulk constant $\alpha_{0}$ is smaller than the total Gilbert damping $\alpha=\alpha_{0}+\alpha^{\prime}$. The additional damping $\alpha^{\prime}$ caused by the spin pumping is observable in, for example, FMR spectra and is the main object of interest here.

\section{SPIN-ACCUMULATION-DRIVEN BACKFLOW IN THE $F-N$ AND $N-F-N$ MULTILAYERS}

The precession of the magnetization does not cause any charge current in the system. The spin accumulation or nonequilibrium chemical potential imbalance $\boldsymbol{\mu}_{s}(x)$ [similar to Eq. (4), but spatially dependent now] in the normal metal is a vector, which depends on the distance from the interface $x$, $0<x<L$, where $L$ is the thickness of the normal-metal film, see Fig. 2.

When the ferromagnetic magnetization steadily rotates around the $z$ axis, $\mathbf{m} \times \mathbf{m}$ and the normal-metal spin accumulation $\boldsymbol{\mu}_{s}(x)$ are oriented along $z$, as depicted in Fig. 2. There is no spin imbalance in the ferromagnet, because $\boldsymbol{\mu}_{s}$ is perpendicular to the magnetization direction $\mathbf{m}$. As shown below, the time-dependent $\boldsymbol{\mu}_{s}$ is also perpendicular to $\mathbf{m}$ even in the case of a precessing ferromagnet with time-dependent 
instantaneous rotation axis, as long as the precession frequency $\omega$ is smaller than the spin-flip rate $\tau_{\mathrm{SF}}^{-1}$ in the normal metal.

The spin accumulation diffuses into the normal metal as

$$
i \omega \boldsymbol{\mu}_{s}=D \partial_{x}^{2} \boldsymbol{\mu}_{s}-\tau_{\mathrm{SF}}^{-1} \boldsymbol{\mu}_{s},
$$

where $D$ is the diffusion coefficient. The boundary conditions are determined by the continuity of the spin current from the ferromagnet into the normal metal at $x=0$ and the vanishing of the spin current at the outer boundary $x=L$ :

$$
\begin{gathered}
x=0: \partial_{x} \boldsymbol{\mu}_{s}=-2(\hbar \mathcal{N S} D)^{-1} \mathbf{I}_{s}, \\
x=L: \partial_{x} \boldsymbol{\mu}_{s}=0,
\end{gathered}
$$

where $\mathcal{N}$ is the (one-spin) density of states in the film and $S$ is the area of the interface. The solution to Eq. (8) with the boundary conditions [Eqs. (9)] is

$$
\boldsymbol{\mu}_{S}(x)=\frac{\cosh \kappa(x-L)}{\sinh \kappa L} \frac{2 \mathbf{I}_{s}}{\hbar \mathcal{N S D} \kappa}
$$

with the wave vector $\kappa=\lambda_{\mathrm{SD}}^{-1} \sqrt{1+i \omega \tau_{\mathrm{SF}}}$, where $\lambda_{\mathrm{SD}}$ $\equiv \sqrt{D \tau_{\mathrm{SF}}}$ is the spin-flip diffusion length in the normal metal. In Ref. 22 we used arguments similar to those in the present paper to calculate the spin accumulation (10) generated by the precessing magnetization. While the size of the effect and its relevance for spintronic applications are detailed in Ref. 22 , in this work we focus on the role of the spin accumulation in the dynamics of the ferromagnetic magnetization.

We assume in the following that the precession frequency $\omega$ is smaller than the spin-flip relaxation rate $\omega \ll \tau_{\mathrm{SF}}^{-1}$ so that $\kappa \approx \lambda_{\mathrm{SD}}^{-1}$. For a static applied field of $1 \mathrm{~T}$, typically $\omega$ $\sim 10^{11} \mathrm{~s}^{-1}$. The elastic scattering rate corresponding to a mean free path of $\lambda_{\mathrm{el}} \sim 10 \mathrm{~nm}$ is $\tau_{\mathrm{el}}^{-1} \sim 10^{14} \mathrm{~s}^{-1}$. Consequently, the derivation below is restricted to metals with a ratio of spin-conserved to spin-flip scattering times $\epsilon$ $\equiv \tau_{\mathrm{el}} / \tau_{\mathrm{SF}} \gtrsim 10^{-3}$. In practice, ${ }^{23}$ this condition is easily satisfied with higher impurity atomic numbers $Z$ (as $\epsilon$ scales as ${ }^{24}$ $\left.Z^{4}\right)$. The high-frequency limit $\omega \gtrsim \tau_{s f}^{-1}$, on the other hand, is relevant for hybrids with little spin-flip scattering in the normal metal, and was discussed in the context of the spinbattery concept. ${ }^{22}$ Nevertheless, we will see that a sizable Gilbert damping enhancement requires a large spin-flip probability $\epsilon \gtrsim 10^{-1}$ (thereby guaranteeing that $\omega \ll \tau_{s f}^{-1}$ ) unless the frequency is comparable with the elastic scattering rate in the normal metal. The latter regime will not be treated in this paper.

Using relation $D=v_{F}^{2} \tau_{\mathrm{el}} / 3$ between the diffusion coefficient $D$ (in three dimensions), the Fermi velocity $v_{F}$, and the elastic scattering time $\tau_{\mathrm{el}}$, we find for the spin-diffusion length

$$
\lambda_{\mathrm{SD}}=v_{F} \sqrt{\tau_{\mathrm{el}} \tau_{\mathrm{SF}} / 3}
$$

An effective energy-level spacing of the states participating in the spin-flip scattering events in a thick film can be defined by

$$
\delta_{\mathrm{SD}} \equiv\left(\mathcal{N} S \lambda_{\mathrm{SD}}\right)^{-1}
$$

The spin-accumulation-driven spin current $\mathbf{I}_{s}^{\text {back }}$ through the interface reads ${ }^{25}$

$$
\begin{aligned}
\mathbf{I}_{s}^{\text {back }}= & \frac{1}{8 \pi}\left[2 g_{r}^{\uparrow \downarrow} \boldsymbol{\mu}_{s}(x=0)+2 g_{i}^{\uparrow \downarrow} \mathbf{m} \times \boldsymbol{\mu}_{s}(x=0)\right. \\
& \left.+\left(g^{\uparrow \uparrow}+g^{\downarrow \downarrow}-2 g_{r}^{\uparrow \downarrow}\right)\left[\mathbf{m} \cdot \boldsymbol{\mu}_{s}(x=0)\right] \mathbf{m}\right] .
\end{aligned}
$$

Substituting Eq. (10) into Eq. (13), we find for the total spin current [Eq. (6)]

$$
\begin{aligned}
\mathbf{I}_{s}= & \mathbf{I}_{s}^{\text {pump }}-\frac{\beta}{2}\left[2 g_{r}^{\uparrow \downarrow} \mathbf{I}_{s}+2 g_{i}^{\uparrow \downarrow} \mathbf{m} \times \mathbf{I}_{s}+\left(g^{\uparrow \uparrow}+g^{\downarrow \downarrow}-2 g_{r}^{\uparrow \downarrow}\right)\right. \\
& \left.\times\left(\mathbf{m} \cdot \mathbf{I}_{s}\right) \mathbf{m}\right]
\end{aligned}
$$

where the spin current returning into the ferromagnet is governed by the "backflow" factor $\beta$,

$$
\beta \equiv \frac{\tau_{\mathrm{SF}} \delta_{\mathrm{SD}} / h}{\tanh \left(L / \lambda_{\mathrm{SD}}\right)} .
$$

When the normal metal is shorter than the spin-diffusion length $\left(L \ll \lambda_{\mathrm{SD}}\right), \beta \rightarrow \tau_{\mathrm{SF}} \delta / h$, where $\delta=(\mathcal{N} S L)^{-1}$ is the energy-level splitting. In the opposite regime of thick normal metals $\left(L \gg \lambda_{\mathrm{SD}}\right), \beta \rightarrow \tau_{\mathrm{SF}} \delta_{\mathrm{SD}} / h$. Basically, $\beta$ [Eq. (15)] is therefore the ratio between the energy level spacing of the normal-metal film with a thickness $L_{\mathrm{SF}}=\min \left(L, \lambda_{\mathrm{SD}}\right)$ and the spin-flip rate.

By inverting Eq. (14), we may express the total spin current $\mathbf{I}_{s}$ in terms of the pumped spin current $\mathbf{I}_{s}^{\text {pump }}$ [Eq. (1)]

$$
\mathbf{I}_{s}=\left[1+\beta g_{r}^{\uparrow \downarrow}+\frac{\left(\beta g_{i}^{\uparrow \downarrow}\right)^{2}}{1+\beta g_{r}^{\uparrow \downarrow}}\right]^{-1} \times\left(1-\frac{\beta g_{i}^{\uparrow \downarrow}}{1+\beta g_{r}^{\uparrow \downarrow}} \mathbf{m} \times\right) \mathbf{I}_{s}^{\text {pump }} .
$$

After substituting Eq. (1) into Eq. (16), we recover the form of Eq. (1) for the total spin current $\mathbf{I}_{s}$, but with a redefined spin-pumping conductance $\widetilde{A} \equiv \widetilde{A}_{r}+i \widetilde{A}_{i}$

$$
\mathbf{I}_{s}=\frac{\hbar}{4 \pi}\left(\tilde{A}_{r} \mathbf{m} \times \frac{d \mathbf{m}}{d t}-\tilde{A}_{i} \frac{d \mathbf{m}}{d t}\right) .
$$

$\widetilde{A}$ can be expressed in terms of the mixing conductance $g \uparrow \downarrow$ and the backflow factor $\beta$ by

$$
\begin{aligned}
\left(\begin{array}{c}
\tilde{A}_{r} \\
\widetilde{A}_{i}
\end{array}\right)= & \left(\begin{array}{cc}
1 & \beta g_{i}^{\uparrow \downarrow}\left(1+\beta g_{r}^{\uparrow \downarrow}\right)^{-1} \\
-\beta g_{i}^{\uparrow \downarrow}\left(1+\beta g_{r}^{\uparrow \downarrow}\right)^{-1} & 1
\end{array}\right) \\
& \times\left[1+\beta g_{r}^{\uparrow \downarrow}+\frac{\left(\beta g_{i}^{\uparrow \downarrow}\right)^{2}}{1+\beta g_{r}^{\uparrow \downarrow}}\right]^{-1}\left(\begin{array}{c}
g_{r}^{\uparrow \downarrow} \\
g_{i}^{\uparrow \downarrow}
\end{array}\right) .
\end{aligned}
$$

It has been shown ${ }^{26}$ that for realistic $F-N$ interfaces $g_{i}^{\uparrow \downarrow}$ $\ll g_{r}^{\uparrow \downarrow}$, so that $g^{\uparrow \downarrow} \approx g_{r}^{\uparrow \downarrow}$. (The latter approximation will be implied for the rest of the paper.) In this important regime, $\widetilde{A}_{i}$ vanishes and the term proportional to $\widetilde{A}_{r}$ in Eq. (17) has the same form as and therefore enhances the phenomenological Gilbert damping. This can be easily seen after substituting Eq. (17) into Eq. (7): The last term on the right-hand side of 
Eq. (7) can be combined with the second term by defining the total Gilbert damping coefficient $\alpha=\alpha_{0}+\alpha^{\prime}$, where

$$
\alpha^{\prime}=\left[1+g^{\uparrow \downarrow} \frac{\tau_{\mathrm{SF}} \delta_{\mathrm{SD}} / h}{\tanh \left(L / \lambda_{\mathrm{SD}}\right)}\right]^{-1} \frac{g_{L} g^{\uparrow \downarrow}}{4 \pi \mu}
$$

is the additional damping constant due to the interfacial $F-N$ coupling. Here, $g_{L}$ is the $g$ factor and $\mu$ is the total film magnetic moment in units of $\mu_{B}$. Equation (19) is the main result of this section. When $L \rightarrow \infty$, Eq. (19) reduces to a simple result: $\alpha^{\prime}=g_{L} g_{\text {eff }}^{\uparrow \downarrow} /(4 \pi \mu)$, where

$$
\frac{1}{g_{\mathrm{eff}}^{\uparrow \downarrow}}=\frac{1}{g^{\uparrow \downarrow}}+R_{\mathrm{SD}}
$$

Here $R_{\mathrm{SD}}=\tau_{\mathrm{SF}} \delta_{\mathrm{SD}} / h$ is the resistance (per spin, in units of $h / e^{2}$ ) of the normal-metal layer of thickness $\lambda_{\mathrm{SD}}$. [Which follows from the Einstein's relation $\sigma=e^{2} D \mathcal{N}$ connecting conductivity $\sigma$ with the diffusion coefficient $D$, and using Eq. (12).] It follows that the effective spin pumping out of the ferromagnet is governed by $g_{\text {eff }}^{\uparrow \downarrow}$, i.e., the conductance of the $F-N$ interface in series with diffusive normal-metal film with thickness $\lambda_{\mathrm{SD}} \cdot{ }^{19}$

The prefactor on the right-hand side of Eq. (19) suppresses the additional Gilbert damping ${ }^{4}$ due to the spin angular momentum that diffuses back into the ferromagnet. It was disregarded in Ref. 4 where the normal metal was viewed as a perfect spin sink. Because spins accumulate in the normal metal perpendicular to the ferromagnetic magnetization, the spin-accumulation-driven transport across the $F-N$ contact, as well as the spin pumping, is governed by a mixing conductance. This explains why the other components of the conductance matrix [Eq. (2)] do not enter Eq. (19).

We now estimate the numerical values of the parameters in Eq. (19) for transition metal ferromagnets $\mathrm{Fe}, \mathrm{Co}$, and $\mathrm{Ni}$, in contact with relatively clean simple normal metals $\mathrm{Al}, \mathrm{Cr}$, $\mathrm{Cu}, \mathrm{Pd}, \mathrm{Ag}, \mathrm{Ta}, \mathrm{Pt}$, and $\mathrm{Au}$. For an isotropic electron gas, $\mathcal{N}=k_{F}^{2} /\left(\pi \hbar v_{F}\right)$. Using Eqs. (11) and (12), we find $h /\left(\delta_{\mathrm{SD}} \tau_{\mathrm{SF}}\right)=4 \sqrt{\epsilon / 3} N_{\mathrm{ch}}$, where $N_{\mathrm{ch}}=S k_{F}^{2} /(4 \pi)$ is the number of transverse channels in the normal metal and $\epsilon$ $\equiv \tau_{\mathrm{el}} / \tau_{\mathrm{SF}}$ is the spin-flip probability at each scattering. In Ref. 26, $g^{\uparrow \downarrow}$ was calculated for $\mathrm{Co}-\mathrm{Cu}$ and $\mathrm{Fe}-\mathrm{Cr}$ interfaces by first-principles band-structure calculations. It was found that irrespective of the interfacial disorder, $g^{\uparrow \downarrow} \approx N_{\mathrm{ch}}$ for these material combinations. As shown in Ref. $19, g^{\uparrow \downarrow}$ has to be renormalized in such limit, making the effective conductances about twice as large. We thus arrive at an estimate

$$
\frac{\alpha_{\infty}^{\prime}}{\alpha^{\prime}} \approx 1+\left[\sqrt{\epsilon} \tanh \left(L / \lambda_{\mathrm{SD}}\right)\right]^{-1},
$$

where $\alpha_{\infty}^{\prime}=g_{L} g^{\uparrow \downarrow} /(4 \pi \mu)$ is the Gilbert damping enhancement assuming infinite spin-flip rate in the normal metal $\tau_{\mathrm{SF}} \rightarrow 0$, i.e., treating it as a perfect spin sink. ${ }^{4}$

It follows that only for a high spin-flip probability $\epsilon$ $\geq 10^{-2}$, the normal-metal film can be a good spin sink so that $\alpha^{\prime} \sim \alpha_{\infty}^{\prime}$. This makes the lighter metals, such as $\mathrm{Al}, \mathrm{Cr}$, and $\mathrm{Cu}$, as well as heavier metals with only $s$ electrons in the conduction band, such as $\mathrm{Ag}, \mathrm{Au}$, and $\mathrm{Ta}$ less effective spin sinks since these metals have a relatively small spin-orbit coupling, typically corresponding to $\epsilon \lessgtr 10^{-2} .{ }^{23,27,28}$ Heavier elements with $Z \gtrsim 50$ and $p$ or $d$ electrons in the conduction band, such as Pd and Pt, on the other hand, can be good or nearly perfect spin sinks as they have a much larger $\epsilon$ $\geq 10^{-1} .{ }^{23}$ This conclusion explains the hierarchy of the observed Gilbert damping enhancement in Ref. 6: Pt has about 2 electrons per atom in the conduction band, which are hybridized with $d$ orbitals, and a large atomic number $Z=78$ and, consequently, leads to a large magnetization damping enhancement in the $N-F-N$ sandwich for thin ferromagnetic films. Pd which is above $\mathrm{Pt}$ in the periodic table having similar atomic configuration but smaller atomic number $Z=46$ leads to a sizable damping, but smaller than for Pt by a factor of 2 . Ta is a heavy element, $Z=73$, but has only $s$ electrons and the damping enhancement is an order of magnitude smaller than in Pt. Finally, $\mathrm{Cu}$ is a relatively light element, $Z=29$, with $s$ electrons only and does not cause an observable damping enhancement at all. According to Eq. (21), a sufficiently thick active layer, $L \gtrsim \lambda_{\mathrm{SD}}$, is also required for a sizable spin relaxation.

The limit of a large ratio of spin-flip to non-spin-flip scattering $\epsilon \sim 1$ deserves special attention. In this regime, Eq. (21) does not hold, since by using the diffusion equation [Eq. (8)] and boundary conditions [Eqs. (9)] we implicitly assumed that $\epsilon \ll 1$. If $\epsilon \gtrsim 10^{-1}$, on the other hand, even interfacial scattering alone can efficiently relax the spin imbalance, and such films, therefore, are good or nearly perfect spin sinks (so that $\alpha^{\prime} \sim \alpha_{\infty}^{\prime}$ ), regardless of their thickness (in particular, they can be thinner than the elastic mean free path).

Infinite vs vanishing spin-flip rates in the normal metal are two extreme regimes for the magnetization dynamics in $F-N$ bilayers. In the former case, the damping constant $\alpha$ $=\alpha_{0}+g_{L} g^{\uparrow \downarrow} /(4 \pi \mu)$ is significantly enhanced for thin ferromagnetic films, whereas in the latter case, $\alpha=\alpha_{0}$ is independent of the ferromagnetic film thickness. Experimentally, the two regimes are accessible by using $\mathrm{Pt}$ as a perfect or $\mathrm{Cu}$ as a poor spin sink in contact with a ferromagnetic thin film, as done in Ref. 6 for $N$-Py- $N$ sandwiches. (Using the $N-F-N$ trilayer simply increases $\alpha^{\prime}$ by the factor of 2, as compared to the $F-N$ bilayer, due to the spin pumping through the two interfaces.) The measured damping parameter $G=\gamma M_{s} \alpha$ is shown in Fig. 3 by circles.

For the $\mathrm{Cu}-\mathrm{Py}-\mathrm{Cu}$ trilayer, our theory predicts $G(d)$ $=G_{0}$, while for the Pt-Py-Pt sandwich

$$
G(d)=G_{0}+\frac{\left(g_{L} \mu_{B}\right)^{2}}{2 \pi \hbar} \frac{g^{\uparrow \downarrow} S^{-1}}{d}
$$

as a function of ferromagnetic film thickness $d$. The Py $g$ factor is $g_{L} \approx 2.1{ }^{6}$ These expression agree with the experiments for $G_{0}=1.0 \times 10^{8} \mathrm{~s}^{-1}$ and $g^{\uparrow \downarrow} S^{-1}=2.6 \times 10^{15} \mathrm{~cm}^{-2}$ (see Fig. 3). Both numbers are very reasonable: $G_{0}$ equals the bulk value $0.7-1.0 \times 10^{8} \mathrm{~s}^{-1}$ for $\mathrm{Py},{ }^{29}$ while $g^{\uparrow \downarrow} S^{-1}$ compares well with $g^{\uparrow \downarrow} S^{-1} \approx 1.6 \times 10^{15} \mathrm{~cm}^{-2}$ found in 


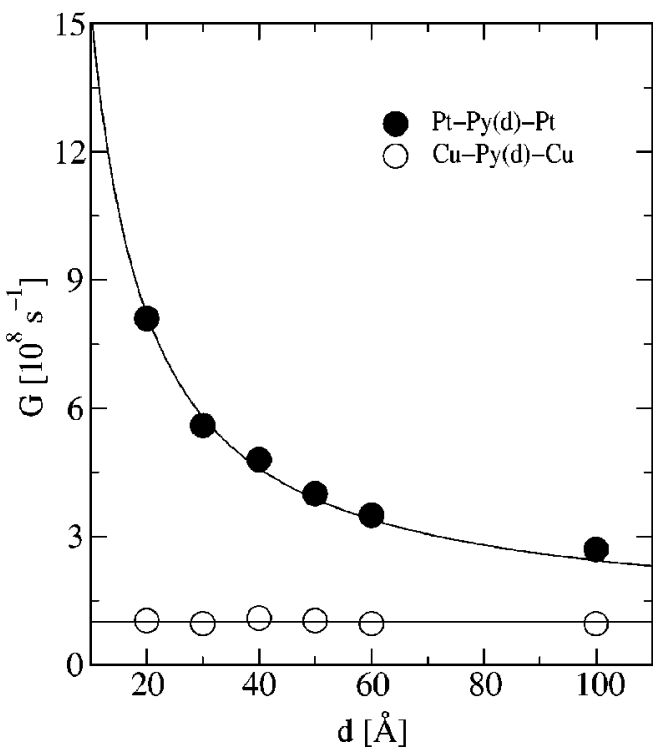

FIG. 3. Circles show measured (Ref. 6) Gilbert parameter $G$ of a permalloy film with thickness $d$ sandwiched between two normalmetal $(\mathrm{Pt}$ or $\mathrm{Cu})$ layers. Solid lines are predictions of our theory with two fitting parameters, $G_{0}$, and $g^{\uparrow \downarrow}$-Py bulk damping and Py-Pt mixing conductance, respectively, see Eq. (22).

angular-magnetoresistance (aMR) measurements in $\mathrm{Py}-\mathrm{Cu}$ hybrids. ${ }^{19}$ (We recall that here one has to use the renormalized mixing conductance $\widetilde{g}^{\uparrow \downarrow}$, in the notation of Ref. 19.) In fact, since Pt has two conduction electrons per atom, while $\mathrm{Cu}$-only one, and they have similar crystal structures, we expect $g^{\uparrow \downarrow}$ to be larger in the case of the Py-Pt hybrid, justifying the value used to fit the experimental data. We have thus demonstrated that the additional damping in ferromagnetic thin films can be used to measure the mixing conductance of the $F-N$ interface.

\section{MAGNETIC DAMPING IN $F-N 1-N 2$ TRILAYER}

In this section we consider ferromagnetic spin pumping into a bilayer N1-N2 normal-metal system, see Fig. 4. It is

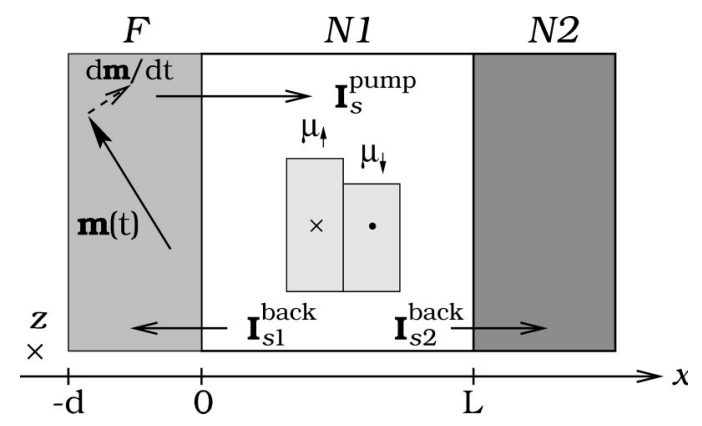

FIG. 4. Same as Fig. 2, but now the normal-metal system is composed of a bilayer N1-N2. Ferromagnetic precession pumps spins into the first normal-metal layer $N 1$. The spin build-up in $N 1$ may flow back into the ferromagnet $F$ as spin current $\mathbf{I}_{s 1}^{\text {back }}$, relax in $N 1$, or return to the second normal-metal layer $N 2$ as spin current $\mathbf{I}_{s 2}^{\text {back }}$. The spin accumulation in $N 2$ is disregarded since the layer is assumed to be a perfect spin sink.

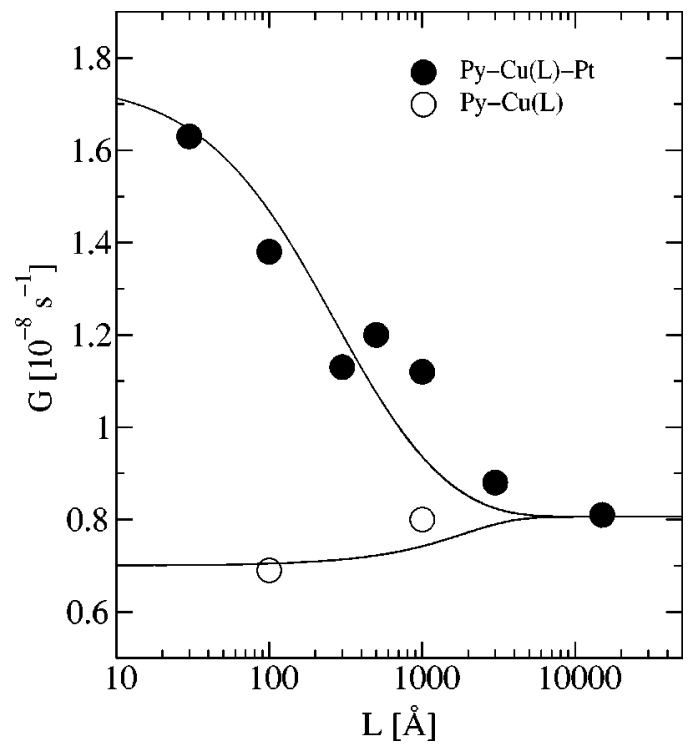

FIG. 5. Circles show the measurements by Mizukami et al. (Ref. 16) of the Gilbert damping in $\mathrm{Py}-\mathrm{Cu}-\mathrm{Pt}$ trilayer and $\mathrm{Py}-\mathrm{Cu}$ bilayer as a function of the $\mathrm{Cu}$ buffer thickness $L$. Solid lines are our theoretical prediction according to Eqs. (26) and (27).

assumed that the spins are driven into the first normal-metal film (N1) of thickness $L$. While in $N 1$, spins are allowed to diffuse through the film, where they can relax, diffuse back into the ferromagnet, or reach the second normal-metal layer (N2). N2 is taken to be a perfect spin sink: spins reaching $N 2$ either relax immediately by spin-flip processes or are carried away before diffusing back into $N 1$. We show that measuring the ferromagnetic magnetization damping as a function of $L$ in this configuration can be used to study the dc mixing conductance of the two $N 1$ film interfaces as well as the $N 1$ spin-diffusion time.

The analysis in this section was inspired by experiments of Mizukami et al., ${ }^{16}$ who in a follow-up to their systematic study of Gilbert damping in $N-\mathrm{Py}-N$ sandwiches, ${ }^{6}$ studied magnetization damping in $\mathrm{Py}-\mathrm{Cu}$ and $\mathrm{Py}-\mathrm{Cu}-\mathrm{Pt}$ hybrids as a function of $\mathrm{Cu}$ film thickness $L$. The measured damping parameter $G$ is shown by circles in Fig. 5. As shown in the preceding section, $\mathrm{Cu}$ is a poor sink for the pumped spins, while Pt is nearly a perfect spin absorber, thus identifying the $\mathrm{Cu}$ film with $N 1$ and the Pt layer with $N 2$.

We use the same notation as in the previous section to discuss the $F-N 1$ spin pumping with subsequent spin diffusion through $N 1$. Similar to Eqs. (9), the boundary conditions for the diffusion equation (8) in the normal metal $N 1$ are now

$$
\begin{aligned}
& x=0: \quad \partial_{x} \boldsymbol{\mu}_{s}=-2(\hbar \mathcal{N S} D)^{-1} \mathbf{I}_{s 1}, \\
& x=L: \quad \partial_{x} \boldsymbol{\mu}_{s}=-2(\hbar \mathcal{N} S D)^{-1} \mathbf{I}_{s 2} .
\end{aligned}
$$

$\mathbf{I}_{s 1}$ and $\mathbf{I}_{s 2}$ are the total spin currents through the left ( $x$ $=0)$ and right $(x=L)$ interfaces, respectively. $\mathbf{I}_{s 1}$ (similarly to $\mathbf{I}_{s}[\mathrm{Eq} .(6)]$ in the previous section) includes the pumped spin current [Eq. (1)] and the spin-accumulation-driven spin 
current [Eq. (13)] contributions. $\mathbf{I}_{s 2}$, on the other hand, is entirely governed by the $N 1 \rightarrow N 2$ spin-accumulation-driven flow

$$
\mathbf{I}_{s 2}=\frac{g}{4 \pi} \boldsymbol{\mu}_{s}(x=L),
$$

where $g$ is the conductance per spin of the $N 1-N 2$ interface.

Solving the diffusion equation (8) with the boundary conditions (23), we find the spin current $\mathbf{I}_{s 1}$ as we did in the preceding section. The Gilbert damping enhancement due to the spin relaxation in the composite normal-metal system is then given by

$$
\alpha^{\prime}=\left[1+g^{\uparrow \downarrow} \frac{\tau_{\mathrm{SF}} \delta_{\mathrm{SD}}}{h} \frac{1+\tanh \left(L / \lambda_{\mathrm{SD}}\right) g \tau_{\mathrm{SF}} \delta_{\mathrm{SD}} / h}{\tanh \left(L / \lambda_{\mathrm{SD}}\right)+g \tau_{\mathrm{SF}} \delta_{\mathrm{SD}} / h}\right]^{-1} \frac{g_{L} g^{\uparrow \downarrow}}{4 \pi \mu} .
$$

Setting $g=0$ decouples the two normal-metal systems and reduces Eq. (25) to Eq. (19) giving the damping coefficient of the $F-N 1$ bilayer. From Eq. (25), we get for the $\mathrm{Py}-\mathrm{Cu}$ (L)-Pt trilayer

$$
\begin{aligned}
G(L)= & G_{0}+\left[1+g^{\uparrow \downarrow} \frac{\tau_{\mathrm{SF}} \delta_{\mathrm{SD}}}{h} \frac{1+\tanh \left(L / \lambda_{\mathrm{SD}}\right) g \tau_{\mathrm{SF}} \delta_{\mathrm{SD}} / h}{\tanh \left(L / \lambda_{\mathrm{SD}}\right)+g \tau_{\mathrm{SF}} \delta_{\mathrm{SD}} / h}\right]^{-1} \\
& \times \frac{\left(g_{L} \mu_{B}\right)^{2}}{2 h} \frac{g^{\uparrow \downarrow} S^{-1}}{d}
\end{aligned}
$$

and for the $\mathrm{Py}-\mathrm{Cu}(L)$ bilayer (putting $g=0$ )

$$
G(L)=G_{0}+\left[1+\frac{g^{\uparrow \downarrow} \tau_{\mathrm{SF}} \delta_{\mathrm{SD}} / h}{\tanh \left(L / \lambda_{\mathrm{SD}}\right)}\right]^{-1} \frac{\left(g_{L} \mu_{B}\right)^{2}}{2 h} \frac{g^{\uparrow \downarrow} S^{-1}}{d} .
$$

In the experiments, the permalloy thickness $d=30 \AA$ is fixed and the $\mathrm{Cu}$ film thickness $L$ is varied between 3 and $1500 \mathrm{~nm}$ as shown by the circles in Fig. 5. Our theoretical results, Eqs. (26) and (27), are plotted in Fig. 5 by solid lines. We use the following parameters: The bulk damping ${ }^{29} \quad G_{0}=0.7$ $\times 10^{8} \mathrm{~s}^{-1}$; the spin-flip probability $\epsilon=1 / 700$ and the spindiffusion length $\lambda_{\mathrm{SD}}=250 \mathrm{~nm}$ for $\mathrm{Cu}$ (which correspond to elastic mean free path $\lambda_{\mathrm{el}}=\sqrt{3 \epsilon} \lambda_{\mathrm{SD}}=16 \mathrm{~nm}$ ), in agreement with values reported in literature; $;^{23,28,30} \quad g^{\uparrow \downarrow} S^{-1}=1.6$ $\times 10^{15} \mathrm{~cm}^{-2}$ from the aMR measurements; ${ }^{19}$ and $\mathrm{gS}^{-1}$ $=3.5 \times 10^{15} \mathrm{~cm}^{-2}$ for the Cu-Pt contact, which lies between values for the majority and minority carriers as measured and calculated $^{31}$ for the $\mathrm{Cu}$-Co interface. Figure 5 shows a remarkable agreement (within the experimental error) between the measurements and our theory. It is important to stress that while the profiles of the trends displayed in Fig. 5 reveal the diffusive nature of spin transfer in the $\mathrm{Cu}$ spacer, they cannot be used to judge the validity of a detailed mechanism for spin injection (relaxation) at the $\mathrm{Py}-\mathrm{Cu}(\mathrm{Cu}-\mathrm{Pt})$ interface. The case of our spin pumping picture is strongly supported by the normalization of the curves (in agreement with experiment), which are governed in our theory by quantities known from other sources.

The trends in Fig. 5 can be understood as follows. Since $\mathrm{Cu}$ is a poor spin sink, a Py-Cu contact with a single $\mathrm{Cu}$ film does not lead to a significant damping enhancement. The small spin-flip ratio, $\epsilon \ll 1$, causes most of the spins transferred into the normal-metal layer to be scattered back and relax in the ferromagnet before flipping their direction in the $\mathrm{Cu}$ buffer. This leads only to a small damping enhancement, which saturates at $L \gg \lambda_{\mathrm{SD}}$ and vanishes in the limit $L$ $\ll \lambda_{\mathrm{SD}}$. The situation changes after a Pt film, a very good spin sink, is connected to the bilayer: If the normal-metal layer is smaller than the elastic mean free path, $L \ll \lambda_{\text {el }}$, the spin accumulation is uniform throughout the $\mathrm{Cu}$ buffer. The spin pumping will now be partitioned. A fraction of the pumped spins reflects back into the ferromagnet, while the rest get transmitted and subsequently relax in the Pt layer. The ratio between these two fractions equals the ratio between the conductance of the $\mathrm{Py}-\mathrm{Cu}$ contact and the Co-Pt contact, $g^{\uparrow \downarrow} / g$, and is of the order of unity. This results in a large magnetization damping as a significant portion of the spin pumping relaxes by spin-orbit scattering in Pt. When $L$ is increased, less spins manage to diffuse through the entire $\mathrm{Cu}$ buffer, and, in the limit $L \gg \lambda_{\mathrm{SD}}$, the majority of the spins scatter back into the ferromagnet or relax in $\mathrm{Cu}$ not feeling the presence of the Pt layer at all. In the intermediate regime, the spin pumping into the Pt layer has an algebraic fall-off on the scale of the elastic mean free path and exponential one on the scale of the spin-diffusion length.

It is important to emphasize that the strong dependence of damping on the $\mathrm{Cu}$ layer thickness $L$ in the Py-Cu-Pt configuration gives evidence of the spin accumulation in the normal-metal system. This spin accumulation, in turn, indicates that an excited ferromagnet (as in the FMR experiment discussed here) transfers spins into adjacent nonmagnetic layers, confirming our claim. ${ }^{4}$ Furthermore, this supports our concept of the spin battery. ${ }^{22}$

Before ending this section, it is illuminating to make a small digression and further study Eq. (26) in the limit of vanishing spin-flip processes in the buffer layer $N 1$. Recalling our definitions for $\lambda_{\mathrm{SD}}$ and $\delta_{\mathrm{SD}}$ [Eqs. (11) and (12)] and taking limit $\tau_{\mathrm{SF}} \rightarrow \infty$, we find that Eq. (26) reduces to Eq. (22), only with $g^{\uparrow \downarrow}$ replaced by $g_{\text {eff }}^{\uparrow \downarrow}$ [similarly to Eq. (20)]:

$$
\frac{1}{g_{\text {eff }}^{\uparrow \downarrow}}=\frac{1}{g^{\uparrow \downarrow}}+R_{N 1}+\frac{1}{g}
$$

where $R_{N 1}$ is the resistance of the $N 1$ layer. The right-hand side of Eq. (28) is simply the inverse mixing conductance of the $N 1$ buffer in series with its two interfaces (one with $F$ and one with $N 2) ;{ }^{19}$ in particular, when layer $N 1$ is thick enough, the total mixing conductance $g_{\text {eff }}^{\uparrow \downarrow}$ is just the conductance of the diffusive normal-metal spacer separating $F$ and $N 2 .^{11,25}$ The spin pumping into layer $N 1$ with the subsequent diffusion and then spin absorption by the ideal spin sink $N 2$ (as discussed in this section) can thus be viewed as the spin pumping across an effective combined scatterer separating the ferromagnet $(F)$ from the perfect spin sink (N2) [as done in obtaining Eq. (22)]. This shows consistency of our approach. 


\section{CONCLUSIONS AND DISCUSSIONS}

Ferromagnets emit a spin current into adjacent normal metals when the magnetization direction changes with time. We recently proposed a novel mechanism for this spin transfer based on the picture of adiabatic spin pumping. ${ }^{4}$ It was shown that our theory explains the increased magnetization damping in ferromagnets in contact with normal metals in measurements of FMR linewidths. ${ }^{6,14,16,17}$

Whereas the spin pumping affects the magnetization dynamics, it also creates a nonequilibrium magnetization in adjacent nonmagnetic films. In this paper we first calculate this spin accumulation for $F-N$ metallic multilayers and find that it induces a spin backflow into the ferromagnetic layer that reduces the spin pumping. This spin-accumulation-driven current is significant for light metals or metals with only $s$ electrons in the conduction band, which have a small spinflip to spin-conserving scattering ratio.

The picture of ferromagnetic spin pumping and subsequent spin diffusion in the adjacent normal-metal layers is also applied to the $F-N 1-N 2$ configuration in order to analyze recent experiments ${ }^{16}$ on magnetization damping in Py$\mathrm{Cu}-\mathrm{Pt}$ trilayers. We showed that our theory quantitatively explains the experimental findings. Our analysis of the experiments by Mizukami et al. ${ }^{6,16}$ shows that FMR of ultrathin ferromagnetic films in contact with single or composite normal-metal buffers is a powerful tool to investigate interfacial transport properties of magnetic multilayers as well as the spin relaxation parameters of the normal metal layers.

\section{ACKNOWLEDGMENTS}

We are grateful to B. I. Halperin and Yu. V. Nazarov for stimulating discussions. This work was supported in part by the DARPA Grant No. MDA 972-01-1-0024, the NEDO International Joint Research Grant Program "Nanomagnetoelectronics," NSF Grant No. DMR 99-81283, the FOM, and the Schlumberger Foundation.

\section{APPENDIX: ADIABATIC SPIN PUMPING}

Here we present a detailed discussion of spin pumping into normal-metal layers by a precessing magnetization direction $\mathbf{m}$ of an adjacent ferromagnet. A schematic of the model is displayed in Fig. 6. The ferromagnetic layer $F$ is a a spin-dependent scatterer that governs electron transport between two [left $(L)$ and right $(R)$ ] normal-metal reservoirs.

The $2 \times 2$ operator $\hat{I}_{l}$ for the charge and spin current in the $l$ th lead $(l=L, R)$ can be expressed in terms of operators $a_{\alpha m, l}(E)\left[b_{\alpha m, l}(E)\right]$ that annihilate a spin- $\alpha$ electron with energy $E$ leaving [entering] the $l$ th lead through the $m$ th channel:

$$
\begin{aligned}
\hat{I}_{l}^{\alpha \beta}(t)= & \frac{e}{h} \sum_{m} \int d E d E^{\prime} e^{i\left(E-E^{\prime}\right) t / \hbar} \\
& \times\left[a_{\beta m, l}^{\dagger}(E) a_{\alpha m, l}\left(E^{\prime}\right)-b_{\beta m, l}^{\dagger}(E) b_{\alpha m, l}\left(E^{\prime}\right)\right] .
\end{aligned}
$$

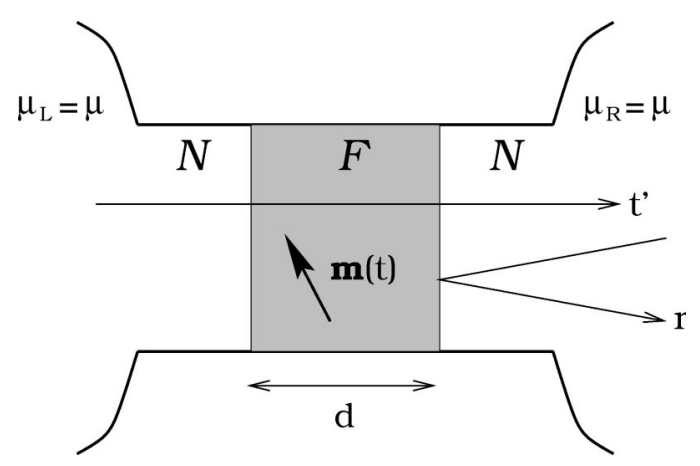

FIG. 6. Ferromagnetic film $(F)$ sandwiched between two normal metal layers $(N)$. The latter are taken to be reservoirs in common thermal equilibrium. The reflection and transmission amplitudes $r$ and $t^{\prime}$ shown here govern the spin current pumped into the right lead.

When the scattering matrix $\hat{s}_{m n, l l^{\prime}}^{\alpha \beta}(t)$ of the ferromagnetic layer varies slowly on the time scales of electronic relaxation in the system, an adiabatic approximation may be used. The annihilation operators for particles entering the reservoirs are then related to the operators of the outgoing states by the instantaneous value of the scattering matrix $b_{\alpha m, l}(E)$ $=\hat{s}_{m n, l l^{\prime}}^{\alpha \beta}(t) a_{\beta n, l^{\prime}}(E)$. In terms of $a_{\alpha m, l}$ only, we can evaluate the expectation value $\left\langle\hat{I}_{l}^{\alpha \beta}(t)\right\rangle$ of the current operator using $\left\langle a_{\alpha m, l}^{\dagger}(E) a_{\beta n, l^{\prime}}\left(E^{\prime}\right)\right\rangle=f_{l}(E) \delta_{\alpha \beta} \delta_{m n} \delta_{l l^{\prime}} \delta\left(E-E^{\prime}\right)$, where $f_{l}(E)$ is the (isotropic) distribution function in the $l$ th reservoir. When the scattering matrix depends on a single time-dependent parameter $X(t)$, then the Fourier transform of the current expectation value $\hat{I}_{l}(\omega)=\int d t e^{i \omega t} \hat{I}_{l}(t)$ can be written as

$$
\hat{I}_{l}(\omega)=\hat{g}_{X, l}(\omega) X(\omega)
$$

in terms of a frequency $\omega$ - and $X$-dependent parameter $\hat{g}_{X, l}: 32$

$$
\begin{aligned}
\hat{g}_{X, l}(\omega)= & -\frac{e \omega}{4 \pi} \sum_{l^{\prime}} \int d E\left(-\frac{\partial f_{l^{\prime}}(E)}{\partial E}\right) \\
& \times \sum_{m n}\left(\frac{\partial \hat{s}_{m n, l l^{\prime}}(E)}{\partial X} \hat{s}_{m n, l l^{\prime}}^{\dagger}(E)-\text { H.c. }\right)
\end{aligned}
$$

Equation (A2) is the first-order (in frequency) correction to the dc Landauer-Büttiker formula. ${ }^{33}$ At equilibrium $f_{R}(E)$ $=f_{L}(E)$, Eq. (A2) is the lowest-order nonvanishing contribution to the current. Furthermore, at sufficiently low temperatures, we can approximate $-\partial f_{l}(E) / \partial E$ by a $\delta$ function centered at Fermi energy. The expectation value of the 2 $\times 2$ particle-number operator $\hat{Q}_{l}(\omega)$ [defined by $\hat{I}_{l}(t)$ $=d \hat{Q}_{l}(t) / d t$ in time or by $\hat{I}_{l}(\omega)=-i \omega \hat{Q}_{l}(\omega)$ in frequency domain] for the $l$ th reservoir is then given by

$$
\hat{Q}_{l}(\omega)=\left(\frac{e}{4 \pi i} \sum_{m n l^{\prime}} \frac{\partial \hat{s}_{m n, l l^{\prime}}}{\partial X} \hat{s}_{m n, l l^{\prime}}^{\dagger}+\text { H.c. }\right) X(\omega),
$$


where the scattering matrices are evaluated at the Fermi energy. Because the prefactor on the right-hand side of Eq. (A4) does not depend on frequency $\omega$, the equation is also valid in time domain. The change in particle number $\delta \hat{Q}_{l}(t)$ is proportional to the modulation $\delta X(t)$ of parameter $X$ and the $2 \times 2$ matrix current (directed into the normal-metal leads) reads

$$
\hat{I}_{l}(t)=e \frac{\partial \hat{n}_{l}}{\partial X} \frac{d X(t)}{d t},
$$

where the "matrix emissivity" into lead $l$ is

$$
\frac{\partial \hat{n}_{l}}{\partial X}=\frac{1}{4 \pi i} \sum_{m n l^{\prime}} \frac{\partial \hat{s}_{m n, l l^{\prime}}}{\partial X} \hat{s}_{m n, l l^{\dagger}}^{\dagger}+\text { H.c. }
$$

If the spin-flip scattering in the ferromagnetic layer is disregarded, the scattering matrix $\hat{s}$ can be written in terms of the spin-up and spin-down scattering coefficients $s^{\uparrow(\downarrow)}$ using the projection matrices $\hat{u}^{\uparrow}=(\hat{1}+\hat{\boldsymbol{\sigma}} \cdot \mathbf{m}) / 2$ and $\hat{u}^{\downarrow}=(\hat{1}-\hat{\boldsymbol{\sigma}}$ $\cdot \mathbf{m}) / 2:^{11}$

$$
\hat{s}_{m n, l l^{\prime}}=s_{m n, l l l^{\prime}}^{\uparrow} \hat{u}^{\uparrow}+s_{m n, l l^{\prime}}^{\downarrow} \hat{u}^{\downarrow} .
$$

The spin current pumped by the magnetization precession is obtained by identifying $X(t)=\varphi(t)$, where $\varphi$ is the azimuthal angle of the magnetization direction in the plane perpendicular to the precession axis. For simplicity, we assume that the magnetization rotates around the $y$ axis: $\mathbf{m}$ $=(\sin \varphi, 0, \cos \varphi)$. Using Eq. (A7), it is then easy to calculate the emissivity [Eq. (A6)] for this process:

$$
\frac{\partial \hat{n}_{l}}{\partial \varphi}=-\frac{1}{4 \pi}\left[A_{r} \sigma_{y}+A_{i}\left(\sigma_{x} \cos \varphi-\sigma_{z} \sin \varphi\right)\right],
$$

where $A_{r}\left(A_{i}\right)=\operatorname{Re}(\operatorname{Im})\left[g^{\uparrow \downarrow}-t^{\uparrow \downarrow}\right]$, as explained in Sec. II. Expanding the $2 \times 2$ current into isotropic and traceless components

$$
\hat{I}=\frac{\hat{1}}{2} I_{c}-\frac{e}{\hbar} \hat{\boldsymbol{\sigma}} \cdot \mathbf{I}_{s},
$$

we identify the charge current $I_{c}$ and spin current $\mathbf{I}_{s}$. Comparing Eqs. (A5), (A8), and (A9), we find that the charge current vanishes, $I_{c}=0$, and the spin current

$$
\mathbf{I}_{s}=\left(A_{i} \cos \varphi, A_{r},-A_{i} \sin \varphi\right) \frac{\hbar}{4 \pi} \frac{d \varphi}{d t}
$$

can be rewritten as Eq. (1). Because the spin current transforms as a vector, it is straightforward to show that Eq. (1) is also valid in the case of the general motion of the magnetization direction.

Even though the mathematics of our scattering approach to adiabatic spin pumping is entirely analogous to the charge-pumping theory developed in Ref. 5, there are some striking differences in the physics. In the case of a spinindependent scatterer as in Ref. 5, the average chargepumping current has the same direction in the two leads, by charge conservation: the charge entering the scattering region through either lead must leave it within a period of the external-gate variations. Whereas the particle number of the two reservoirs must (on average) be conserved also here, the total conduction-electron spin angular momentum is not conserved. In fact, as we explained in Ref. 4 for a symmetric system shown in Fig. 6, a precessing ferromagnet loses angular momentum by polarizing adjacent nonmagnetic conductors. In this respect, the phenomenon looks more similar to a spin "well" or "fountain." An excited ferromagnet ejects spins in all directions into adjacent conductors by losing its own angular momentum, rather than transfers ("pumps") spins from one lead to the other. The angular momentum has to be provided, of course, by the applied magnetic field.
*Present address: Department of Physics, Norwegian University of Science and Technology, N-7491 Trondheim, Norway.

${ }^{1}$ M.A.M. Gijs and G.E.W. Bauer, Adv. Phys. 46, 285 (1997); P.M. Levy, Solid State Phys. 47, 367 (1994); E.B. Myers, D.C. Ralph, J.A. Katine, R.N. Louie, and R.A. Buhrman, Science 285, 867 (1999); J.A. Katine, F.J. Albert, R.A. Buhrman, E.B. Myers, and D.C. Ralph, Phys. Rev. Lett. 84, 3149 (2000); J.-E. Wegrowe, D. Kelly, T. Truong, Ph. Guittienne, and J.-Ph. Ansermet, Europhys. Lett. 56, 748 (2001).

${ }^{2}$ J.C. Slonczewski, J. Magn. Magn. Mater. 159, L1 (1996).

${ }^{3}$ J.C. Slonczewski, J. Magn. Magn. Mater. 195, L261 (1999).

${ }^{4}$ Y. Tserkovnyak, A. Brataas, and G.E.W. Bauer, Phys. Rev. Lett. 88, 117601 (2002).

${ }^{5}$ P.W. Brouwer, Phys. Rev. B 58, R10 135 (1998).

${ }^{6}$ S. Mizukami, Y. Ando, and T. Miyazaki, J. Magn. Magn. Mater. 226, 1640 (2001); Jpn. J. Appl. Phys. 40, 580 (2001).

${ }^{7}$ S.M. Bhagat and P. Lubitz, Phys. Rev. B 10, 179 (1974).

${ }^{8}$ R. Kikuchi, J. Appl. Phys. 27, 1352 (1956); Q. Peng and H.N. Bertram, ibid. 81, 4384 (1997); M. Igarashi, K. Yoshiba, and Y. Sugita, ibid. 87, 5672 (2000).
${ }^{9}$ R.H. Silsbee, A. Janossy, and P. Monod, Phys. Rev. B 19, 4382 (1979); H. Hurdequint and G. Dunifer, J. Phys. (Paris), Colloq. 49, C8 1717 (1988).

${ }^{10}$ L. Berger, Phys. Rev. B 54, 9353 (1996).

${ }^{11}$ A. Brataas, Y.V. Nazarov, and G.E.W. Bauer, Phys. Rev. Lett. 84, 2481 (2000).

${ }^{12}$ X. Waintal, E.B. Myers, P.W. Brouwer, and D.C. Ralph, Phys. Rev. B 62, 12317 (2000).

${ }^{13}$ J.-E. Wegrowe, Phys. Rev. B 62, 1067 (2000).

${ }^{14}$ B. Heinrich, K.B. Urquhart, A.S. Arrott, J.F. Cochran, K. Myrtle, and S.T. Purcell, Phys. Rev. Lett. 59, 1756 (1987).

${ }^{15}$ W. Platow, A.N. Anisimov, G.L. Dunifer, M. Farle, and K. Baberschke, Phys. Rev. B 58, 5611 (1998).

${ }^{16}$ S. Mizukami, Y. Ando, and T. Miyazaki, J. Magn. Magn. Mater. 239, 42 (2002).

${ }^{17}$ R. Urban, G. Woltersdorf, and B. Heinrich, Phys. Rev. Lett. 87, 217204 (2001).

${ }^{18}$ B. Heinrich (private communication).

${ }^{19}$ G.E.W. Bauer, Y. Tserkovnyak, D. Huertas-Hernando, and A. 
Brataas, cond-mat/0205453 (unpublished).

${ }^{20}$ M.D. Stiles and A. Zangwill, Phys. Rev. B 66, 014407 (2002).

${ }^{21}$ T.L. Gilbert, Phys. Rev. 100, 1243 (1955); L. D. Landau, E. M. Lifshitz, and L. P. Pitaevski, Statistical Physics, 3rd ed. (Pergamon, Oxford, 1980), Part 2.

${ }^{22}$ A. Brataas, Y. Tserkovnyak, G.E.W. Bauer, and B.I. Halperin, Phys. Rev. B 66, 060404(R) (2002).

${ }^{23}$ R. Meservey and P.M. Tedrow, Phys. Rev. Lett. 41, 805 (1978).

${ }^{24}$ A.A. Abrikosov and L.P. Gor'kov, Zh. Éksp. Teor. Fiz. 42, 1088 (1962) [Sov. Phys. JETP 15, 752 (1962)].

${ }^{25}$ A. Brataas, Y.V. Nazarov, and G.E.W. Bauer, Eur. Phys. J. B 22, 99 (2001).

${ }^{26}$ K. Xia, P.J. Kelly, G.E.W. Bauer, A. Brataas, and I. Turek, Phys. Rev. B 65, 220401(R) (2002).

${ }^{27}$ G. Bergmann, Z. Phys. B: Condens. Matter 48, 5 (1982); M.
Johnson, Phys. Rev. Lett. 70, 2142 (1993).

${ }^{28}$ Q. Yang, P. Holody, S.-F. Lee, L.L. Henry, R. Loloee, P.A. Schroeder, W.P. Pratt, and J. Bass, Phys. Rev. Lett. 72, 3274 (1994).

${ }^{29}$ C.E. Patton, Z. Frait, and C.H. Wilts, J. Appl. Phys. 46, 5002 (1975); D. Bastian and E. Biller, Phys. Status Solidi A 35, 113 (1976).

${ }^{30}$ F.J. Jedema, A.T. Filip, and B.J. van Wees, Nature (London) 410, 345 (2001).

${ }^{31}$ K. Xia, P.J. Kelly, G.E.W. Bauer, I. Turek, J. Kudrnovsky, and V. Drchal, Phys. Rev. B 63, 064407 (2001).

${ }^{32}$ M. Büttiker, H. Thomas, and A. Prêtre, Z. Phys. B: Condens. Matter 94, 133 (1994).

${ }^{33}$ M. Büttiker, Phys. Rev. Lett. 57, 1761 (1986). 